\title{
Primary HT and risk for breast cancer: a systematic review and meta-analysis
}

\author{
Anna G Angelousi, Valsamo K Anagnostou ${ }^{1}$, Michael K Stamatakos ${ }^{2}$, Georgios A Georgiopoulos ${ }^{3}$ and \\ Konstantinos C Kontzoglou ${ }^{4}$ \\ Department of Endocrinology, University Hospital of Nancy, 13 Rue de Stanislas, Nancy 54000, France, ${ }^{1}$ Department of Internal Medicine, Yale New \\ Haven Hospital, New Haven, Connecticut, USA, ${ }^{2}$ Department of Surgery, Athinaion Hospital, Athens, Greece, ${ }^{3}$ Vascular Laboratory, Department of \\ Therapeutics, Alexandra Hospital, Athens University Medical School, Athens, Greece and ${ }^{4} 2$ nd Department of Surgery, Athens Medical School, Laiko \\ Hospital, Athens, Greece \\ (Correspondence should be addressed to A G Angelousi; Email: a.angelousi@gmail.com)
}

\begin{abstract}
Objective: The association between hypothyroidism and breast cancer has been described from very early on. Breast and thyroid tissue are interconnected on a molecular level mainly through activation of thyroid hormone receptors expressed on cells of the mammary gland as well as on the plasma membrane of breast cancer cells. Despite the experimental evidence the true value of hypothyroidism as a risk factor for breast cancer remains controversial.

Methods: We searched the PubMed database through February 2011 to identify studies that evaluated the association between hypothyroidism and risk for breast cancer as well as the effect of thyroid hormone replacement therapy on breast cancer incidence.

Results: A meta-analysis performed in 12 studies showed that hypothyroidism was not associated with risk for breast cancer (pooled risk ratio $(\mathrm{RR})=1.06,95 \%$ confidence intervals (CIs) 0.82-1.35, $P=0.672$ ). The effect of treatment was assessed in seven studies and no evidence for an association between thyroid hormone replacement and breast cancer was observed with an overall RR of 0.99 (95\% CI $0.73-1.35, P=0.965)$.

Conclusions: Our meta-analysis showed that hypothyroidism is not associated with increased risk for breast cancer and thyroid hormone replacement therapy does not reduce breast cancer prevalence; however, the heterogeneity of the studies analyzed precludes firm conclusions.
\end{abstract}

European Journal of Endocrinology 166 373-381

\section{Introduction}

Breast cancer is the most common type of cancer in women with a cumulative incidence of 182460 new cases per year in the United States, 45822 in the United Kingdom, and 21200 in Canada (1, 2, 3). The association between breast cancer and thyroid gland function has been reported as early as 1896 when Beatson used thyroid extract as a potent treatment for breast cancer (4). In the 1950s, Loeser and Ellerker reported that breast cancer rarely occurred in hyperthyroid women whereas more frequently than expected in hypothyroid women $(5,6)$. Multiple studies have shown that hyperthyroidism may be protective against breast cancer $(7,8)$, while other studies have reported a strong relationship between antithyroid peroxidase autoantibodies (TPO Abs) with the risk for breast cancer $(9,10,11)$.

The thyroid gland and the mammary gland share common physiology; both thyroid follicular and lactating breast cells store iodine by a membrane active transport mechanism known as natrium-iodide symporter (NIS) mediated iodine uptake $(11,12,13,14,15,16,17,18)$ and iodine oxidization in the alveolar mammary cells is carried out by lactohyperoxidase, which is immunologically similar to the thyroid gland's hyperoxidase (16). The crosstalk between the thyroid and the mammary gland mainly involves the triiodothyronine $\left(\mathrm{T}_{3}\right)$ pathway, and activation of thyroid hormone receptors (TR) of the mammary gland induces differentiation and lobular growth in an estrogen-like manner $(19,20)$.

Furthermore, iodine has been shown to play an antioxidant role and thus inhibit breast cancer progression $(11,17,18)$. Overexpression of NIS has been found in malignant breast cells leading to an increased iodine uptake in the mammary gland, playing a protective role for malignant transformation (11). TR gene transcription deregulation has been implicated in breast carcinogenesis (19) and TR- $\alpha$ receptors have been shown to translocate from the nucleus to the cytoplasm of breast cancer cells, suggesting a possible role in the histological changes of the epithelium during 
carcinogenesis (20). It has been shown that hypothyroidism may result in hypersensitization of the mammary glandular epithelium to prolactin and estrogen, thus promoting breast cancer growth (21). Moreover, estrogens as well as pregnancy increase thyroxinebinding globulin concentration that in turns increases serum concentration of total thyroxine $\left(\mathrm{T}_{4}\right)$, with a transient reduction of free $\mathrm{T}_{4}$, and an increase of thyrotropin (TSH) $(22,23)$.

Despite the promising nature of experimental data, hypothyroidism has been investigated as a potential predictor for breast cancer risk with conflicting results $(18,24,25,26,27,28,29,30,31,32,33,34)$. In this systematic review and meta-analysis we sought to determine the association between hypothyroidism and breast cancer as well as the role of standard replacement therapy in modifying the incidence of breast cancer.

\section{Materials and methods}

\section{Data source and search strategy}

We searched the PubMed database on February 20, 2011 to identify studies that evaluated the association of hypothyroidism and breast cancer using the following algorithm: (((hypothyroidism) OR (thyroid dysfunction) OR (thyroid)) AND ((breast cancer) OR (breast neoplasm) OR (breast carcinoma) OR (breast tumor))).

Our search was restricted to English and French literature. One reviewer (A G Angelousi) reviewed the title and abstract to identify studies likely to report associations between hypothyroidism and breast cancer and subsequently obtained the full texts. Additional relevant publications were identified from references of the initially retrieved articles.

\section{Study selection}

The selection of the eligible studies for inclusion in the review was based on the following criteria: retrospective or prospective cohort design or case-control studies with clearly defined source population, clear statement of primary hypothyroidism, statistical methodology including univariate or multivariate analysis and reporting of the resultant odd ratios (ORs) and their 95\% confidence intervals (CIs). We excluded case reports, reviews, commentaries, letters, studies which studied drug-induced hypothyroidism as well as studies assessing thyroid dysfunction based on thyroid hormones measurement in women with breast disease with no previous history of hypothyroidism (Fig. 1).

\section{Data extraction and quality assessment}

Two investigators (A G Angelousi and V K Anagnostou) reviewed independently each eligible manuscript and extracted data on general characteristics of the study including first author's name, institution and country of origin, year of publication, study design, sample size, demographics (age, ethnicity, menopausal status, parity, family history of breast cancer, and hormone replacement treatment), and follow-up period (Table 1). The primary outcome of the meta-analysis concerned the association between hypothyroidism and the breast cancer risk; the association between thyroid hormone replacement therapy and the risk for breast cancer was analyzed as a secondary endpoint. The compared groups, the clinical covariates incorporated in multivariable analysis and the outcomes studied are presented in Table 2.

\section{Data synthesis and statistical analysis}

Effect sizes and 95\% CIs were estimated by pooling available data using the STATA (StataCorp, Texas, USA) version 10 Software (35). The heterogeneity among studies was analysed by using the Random effects metaanalysis of Der Simonian and Laird's method. ORs were presented as point estimates with 95\% CI. An observed OR of more than 1 implied a higher risk for the test group relative to the reference group and was considered statistically significant if the 95\% CI did not overlap with $1(P<0.05)$. Inter-study heterogeneity was assessed using the $I^{2}$ index (37).

This systematic review followed the Meta-analysis of Observational Studies in Epidemiology guidelines for reporting meta-analyses of observational studies (38).

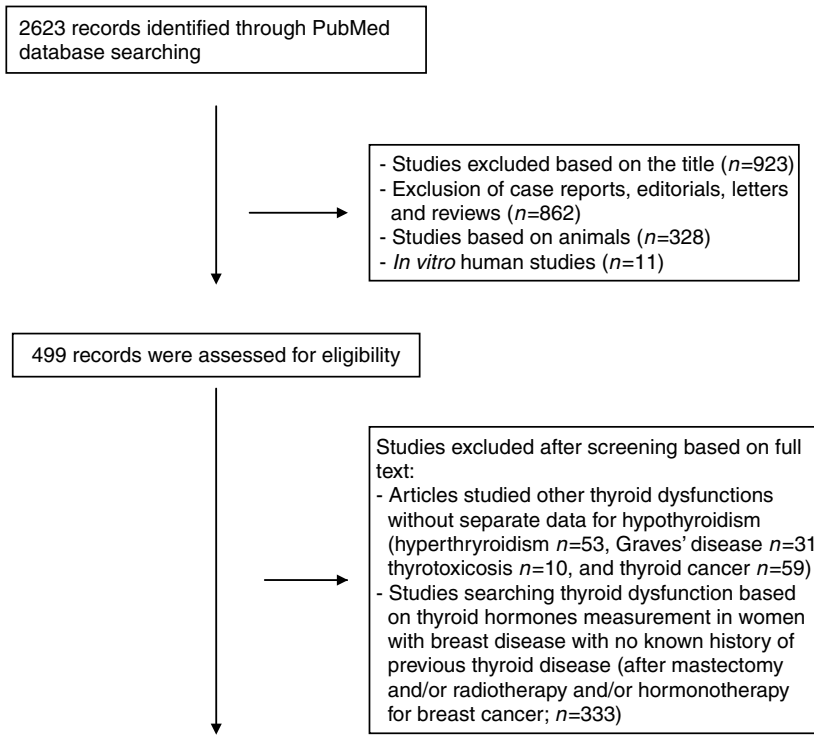

13 studies

Figure 1 Flow diagram showing the selection process for the studies included in the meta-analysis. 


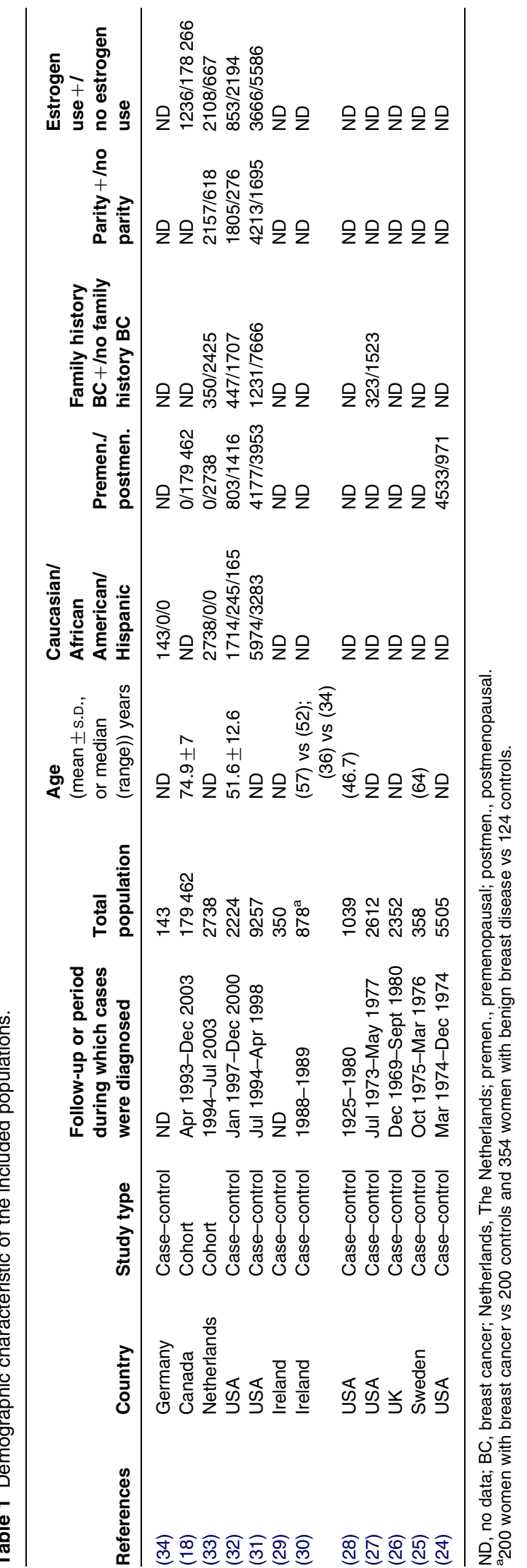

\section{Results}

\section{Identified studies}

A total of 2623 studies were retrieved: of these, 499 studies were selected for further evaluation. Studies evaluating thyroid dysfunction without separate analysis for hypothyroidism $(n=153)$ and studies assessing thyroid dysfunction in terms of thyroid hormone serum levels in women with breast cancer without history of hypothyroidism $(n=333)$ were excluded (Fig. 1).

Of the remaining 13 studies, one was excluded (7) as it was a sub-analysis of a larger study that met the inclusion criteria (28). Finally, 12 studies comprising cohort studies $(18,33)$, and ten case-control studies $(24,25,26,27,28,29,30,31,32,34)$ were selected for further analysis.

\section{Patient demographics}

Clinical characteristics incorporated in the studies included age, ethnicity, menopausal status, family history, parity, and prior hormone replacement therapy; patient characteristics are summarized in Table 1.

Four studies presented data about the ethnicity of their included population which was largely represented by Caucasian people $(31,32,33,34)$. The total population was represented by 9513 premenopausal vs 188577 postmenopausal women - data available in five studies $(18,24,31,32,33)$, and by 13321 women without family history vs 2351 women with family history of breast cancer - data available in four studies $(27,31,32,33)$.

Estrogen replacement therapy was reported in four studies accounting for 4519 women that received hormone replacement therapy and 186673 that did not $(18,31,32,33)$. Of note, in three of the abovementioned studies hormone replacement treatment was significantly more frequent among the breast cancer group compared with controls $(31,32,33)$.

Effective sample size ranged from 61 to 89731 (total $97477)$, with six studies including $<200$ patients $(25$, $28,29,30,33,34)$, four studies including 200-1500 individuals $(24,26,27,32)$, one including 4575 (31), and one including 89731 individuals (18).

\section{Studied outcome}

Ten studies $(18,24,25,26,27,29,30,31,32,34)$ analyzed the incidence of hypothyroidism in women with breast cancer compared with matched healthy controls or women with benign breast disease $(30,34)$. Additional studied parameters included the breast cancer-specific mortality among women with treated vs untreated hypothyroidism (28), the ORs for the association between self-reported hypothyroidism (treated and untreated) and breast cancer (33) and 


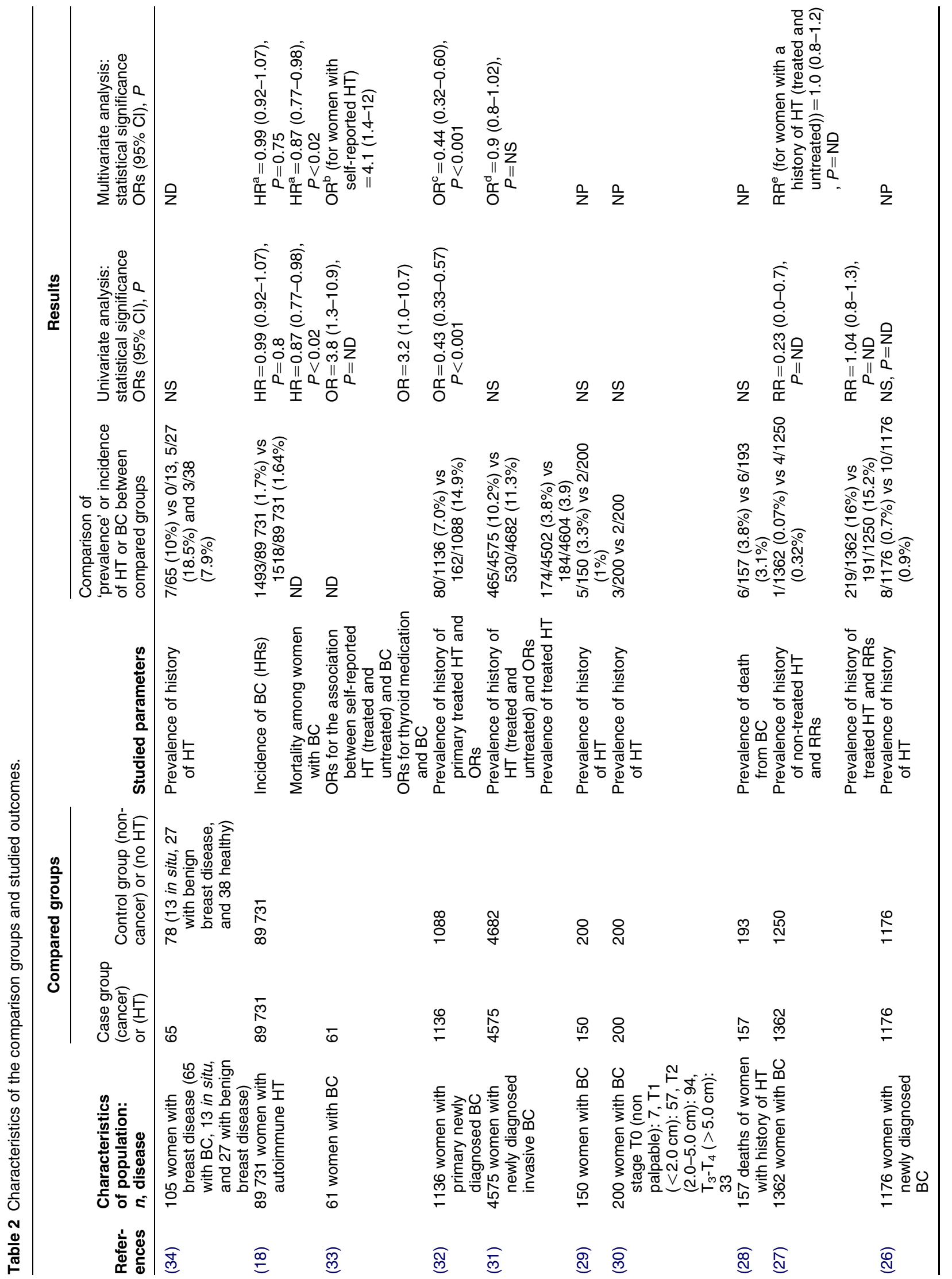




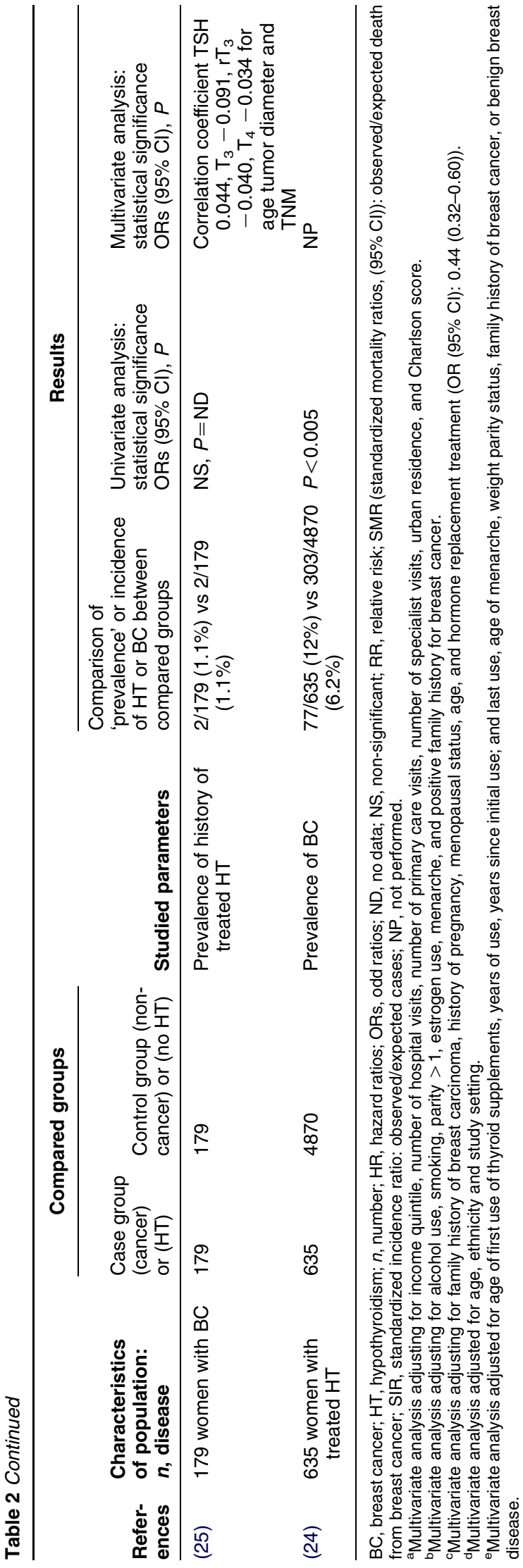

also the survival outcomes for breast cancer in women with hypothyroidism (18) as they presented in Table 2.

Key question 1: does hypothyroidism increase the risk for breast cancer? Eight $(18,25,26,28,29,30$, $31,34)$ studies showed that there is no significant association between primary hypothyroidism and breast cancer. Two of those studies $(18,31)$ incorporated multivariate analysis adjusting for clinical characteristics. Four studies $(24,27,32,33)$ identified a significant association between hypothyroidism and breast cancer in univariate analysis that was subsequently confirmed by multivariate analysis in three of them $(27,32,33)$. Interestingly, one study (33) showed that hypothyroidism was significantly associated with an increased risk for breast cancer as opposed to another study (32) that demonstrated that primary hypothyroidism was a strong protective factor against breast cancer after adjusting for clinical parameters including thyroid hormone replacement therapy. In one study (27), the untreated hypothyroidism was correlated with a significantly lower risk for breast cancer although the incidence of treated hypothyroidism was similar between the women with breast cancer and the women with no breast disease.

Our meta-analysis incorporated all 12 studies; pooled overall risk ratios are shown in Fig. 2. There was a $6 \%$ increase in risk for breast cancer among women with primary hypothyroidism (RR 1.06, 95\% CI 0.82-1.35, $P=0.672$ ); however, the effect did not reach statistical significance due to study heterogeneity. $I^{2}$ values were calculated to quantify heterogeneity between studies, an $I^{2}$ value of $85.2 \%(P<0.001)$ indicated high interstudy heterogeneity. We found no publication bias as indicated by Egger $(P=0.642)$ and Harbord $(P=0.771)$ tests (Fig. 3).

We subsequently conducted two separate analyses stratifying patients by country of origin. In the European group (including six studies with a total population of 6819 , of which 1831 women had breast cancer), a trend toward a significant association between hypothyroidism and higher risk for breast cancer was observed (pooled risk ratio $=1.46,95 \% \mathrm{CI}$ $0.91-2.34, P=0.12) . I^{2}$ was $8 \%$ indicating low interstudy heterogeneity $(P=0.365)$. In the US and Canada groups (including six studies with a total population of 200 099, from which 7073 women had breast cancer and 90523 women had hypothyroidism) no association was identified between hypothyroidism and breast cancer (pooled risk ratio $=0.97,95 \%$ CI $0.73-1.29$, $P=0.837)$; a high inter-study heterogeneity was observed ( $\left.I^{2} 92.4 \%, P<0.001\right)$.

Key question 2: does thyroid hormone replacement therapy affect the risk for breast cancer? Seven studies assessed the association between thyroid hormone replacement treatment and breast cancer 


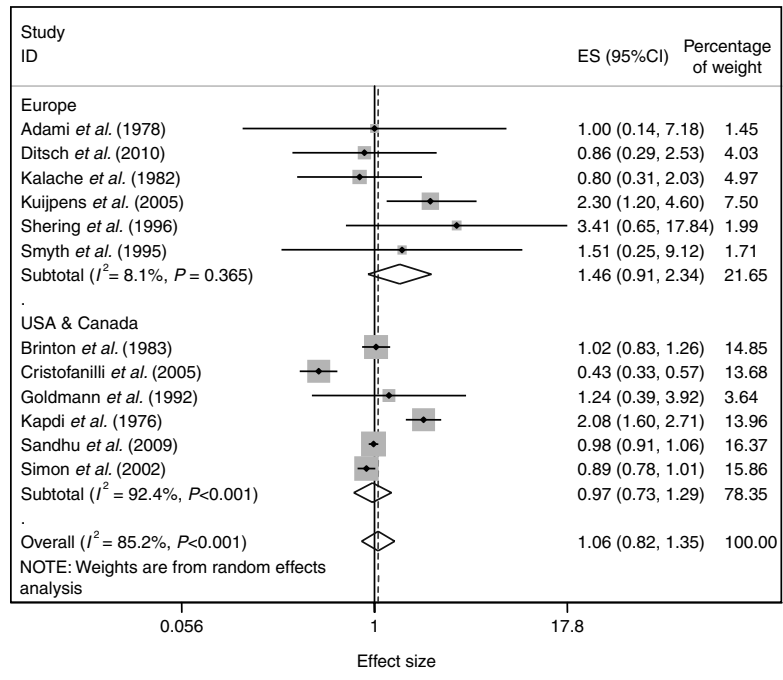

Figure 2 Forest plot. Overall risk ratio for breast cancer in women with primary hypothyroidism in a metanalysis of 12 studies and separate risk ratios for European studies and USA/Canada studies (subtotal effect size). A fixed effects meta-analysis (Mantel-Haenszel method) was performed in the European studies and a random effects meta-analysis in the USA/Canada studies.

risk $(18,24,25,27,31,32,34)$. Of those, five studies failed to identify a difference in risk for breast cancer among women that received thyroid hormone replacement $(18,24,25,27,31)$. One study (34) showed that women who received thyroid hormone replacement treatment were at higher risk for breast cancer; however, results might have been confounded by absence of appropriate matching between the breast cancer and control groups. These findings were consistent with another study (24) that demonstrated that women with treated hypothyroidism had significantly increased risk for breast cancer compared with healthy controls.

We conducted a separate meta-analysis of these seven studies to identify the effect of thyroid replacement therapy on breast cancer risk $(18,24,25,27,31,32$, 34). No statistically significant association between thyroid hormone replacement treatment and breast cancer risk was found (overall pooled risk ratio $=0.99$, 95\% CI 0.73-1.35, $P=0.965)$; however, high interstudy heterogeneity was observed $\left(I^{2}=90.7 \%\right.$, $P<0.001)$. We found no publication bias as indicated by Egger $(P=0.979)$ and Harbord $(P=0.910)$ tests. Results are summarized in Figs 4 and 5.

\section{Discussion}

Our meta-analysis failed to show a statistically significant association between hypothyroidism and risk for breast cancer with a significant heterogeneity observed among studies. Subgroup analysis by country of origin revealed a trend toward increased risk for breast cancer in hypothyroid women in the
European group. Of note, inter-study heterogeneity in the meta-analysis of the six studies of the European group was minimal. We subsequently investigated the potential of thyroid hormone replacement treatment in modifying the risk for breast cancer; the metaanalysis on seven eligible studies failed to reveal a statistically significant effect.

Our findings are not in keeping with a large volume of experimental studies that suggest a strong association between hypothyroidism and breast cancer. Use of elemental iodine $\left(\mathrm{I}_{2}\right)$ has been shown to result in a significant reduction of the growth and number of mammary tumors in experimental animals $(17,39,40)$. Interestingly, altered expression of the $\mathrm{TRb}$ and TRa genes that encode the TRs has been observed in breast cancer patients suggesting that deregulation of thyroid hormone target genes may be involved in breast carcinogenesis (41).

We observed a high inter-study heterogeneity: study design, retrospective nature, and control study group mismatch are major factors that account for the discrepancies seen since the diagnosis of hypothyroidism and replacement treatment specifics were based on patient recall or medical archives, increasing the possibility of misclassification bias. Another contributing factor to the high overall inter-study heterogeneity is the lack of multivariate analysis in seven of the 12 studies $(18,24,26,28,29,30,34)$, as well as the variability of clinical parameters used for adjustment in those studies that performed multivariate analysis. The use of estrogen treatment - a well-known risk factor for breast cancer - was included in a multivariate analysis in only two studies $(32,33)$, as well as the adjustment for family history of breast cancer which was performed in three studies $(31,32,33)$.

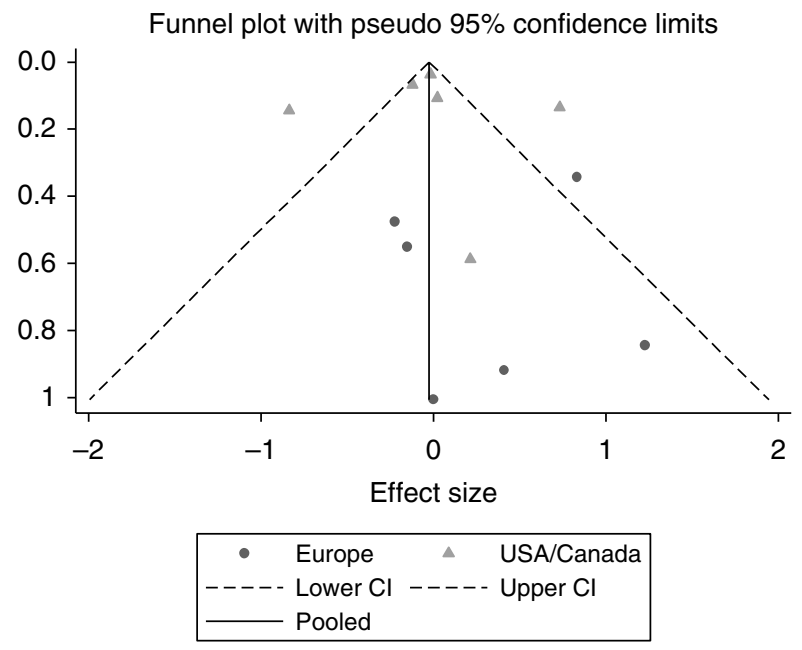

Figure 3 Funnel plot for publication bias of the metaanalysis studying the association of primary hypothyroidism with the risk for breast cancer. Egger $(P$ value $=0.642)$ and Harbord $(P$ value $=0.771)$ tests revealed no publication bias . 


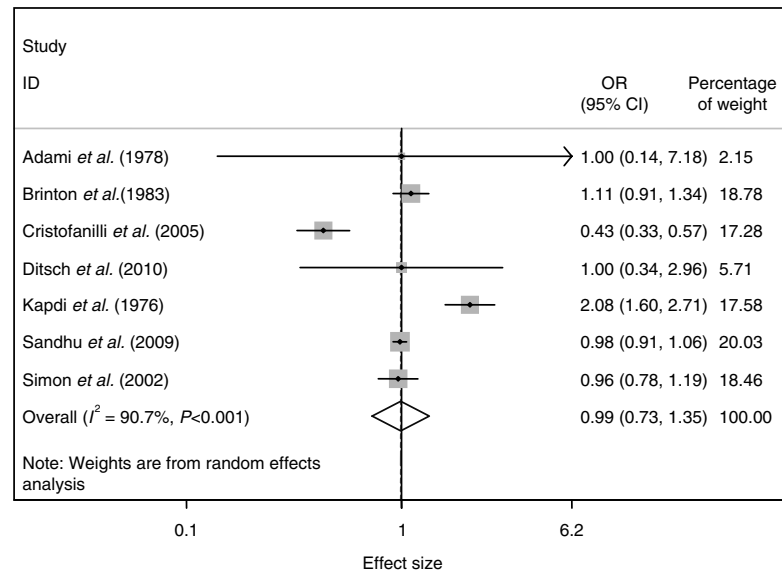

Figure 4 Forest plot. Overall risk ratio for breast cancer in women with thyroid replacement treatment in a meta-analysis of seven studies.

Moreover, few studies incorporated breast cancer stage in subsequent analysis; this is an important limitation since patients with more indolent in situ disease are grouped together with invasive breast cancer. In particular, five studies included data on breast cancer staging $(25,30,32,33,34)$. One of them (32) found that women with primary hypothyroidism were more likely to have localized disease ( 95.0 vs $85.9 \%$ clinical $\mathrm{T} 1$ or $\mathrm{T} 2$ disease, respectively, $P=0.02$ ) and no lymph node involvement ( 62.8 vs $54.4 \%, P=0.15$ ) compared with euthyroid women. These findings were not consistent with the study of Ditsch et al. (34) which observed that the history of hypothyroidism was more frequent although not statistically different in women with breast cancer compared with patients with Tis stage and benign breast disease. Three studies evaluated the association of thyroid hormones circulating levels or thyroid volume with stage at the time of diagnosis independent of the history of hypothyroidism $(25,30,33)$.

To minimize heterogeneity we performed subgroup analysis by country of origin, which revealed a marginal association between hypothyroidism and increased risk for breast cancer in the European group compared with the USA and Canada group. To note that the two groups differ significantly in sample size, with a total population of 6819 vs 200099 for the European and the US groups respectively, in the number of women with treated hypothyroidism (11 vs 2042 for the European and the US groups respectively) as well as in the performance of the multivariate analysis (one study (33) vs four studies $(18,27,31,32)$ performed multivariate analysis in the European and the US groups respectively). Additional meta-regression analysis and comparison of demographics (ethnicity, family history of breast cancer, parity, estrogen replacement treatment, and menopausal status) between the two groups was not feasible since relevant data were not available.

Interestingly, the high inter-study heterogeneity observed was largely caused by two studies $(24,32)$; when these studies were excluded overall heterogeneity was minimized (data not shown). One of them (32) was the only study reporting a statistically significant protective role for hypothyroidism with an objective risk for breast cancer of 0.43; these findings were not replicated in subsequent trials. With respect to the other study (24), the generalizability of its results may be limited by inappropriate matching of the compared groups since the control group (women with no thyroid disease and no thyroid treatment) largely consists of premenopausal (4533 vs 971) and nulliparous women (4349 nulliparous vs 1156 parous) compared with the hypothyroid group.

In this meta-analysis we did not manage to study separately the association of autoimmune hypothyroidism with the risk for breast cancer although it is largely presented in the literature $(9,10,11,15)$ due to the lack of information concerning the thyroid function of the patients with known Hashimoto's thyroiditis or positive TPO Abs. However, it is worth presenting that two of the studies $(29,33)$ included in the meta-analysis showed that the presence of high levels of TPO Abs were related to an increased risk for breast cancer although in one of them (33) this relationship was not confirmed in the follow-up. To the contrary, three other included studies $(18,31,34)$ found no association between autoimmune hypothyroidism (18) or Hashimoto's thyroiditis (31) or high levels of TPO Abs (34) with the risk for breast cancer. To note that in one study (28), women with Hashimoto's thyroiditis who died from breast cancer had the lowest percent of deaths due to breast cancer in the group with thyroid diseases with no further statistical analysis.

Lastly, our meta-analysis failed to demonstrate a statistically significant role of thyroid hormone replacement therapy in modifying the risk for breast cancer. This finding is in agreement with previous studies (18, $40,42,43)$ showing that thyroid hormone replacement therapy did not affect the risk for breast cancer.

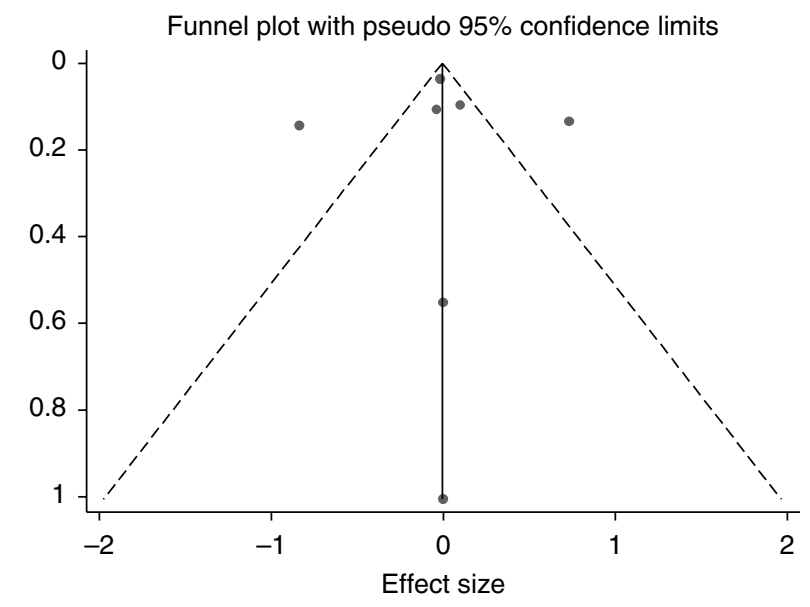

Figure 5 Funnel plot. Publication bias of the meta-analysis of studies presented the association between thyroid hormone replacement treatment and the risk for breast cancer. Egger $(P$ value $=0.979)$ and Harbord $(P$ value $=0.91)$ tests reveal no publication bias. 


\section{Conclusions}

Our meta-analysis failed to demonstrate an association between hypothyroidism and risk for breast cancer; our study was limited by significant inter-study heterogeneity introduced by retrospective study design, inappropriately matched control groups, and absence of multivariate analysis in the majority of studies analyzed. Yet, we can equally postulate that hypothyroid women receiving replacement therapy might benefit from tighter health control and lifestyle modifications that could result in early diagnosis and diminution in risk of breast cancer. Thus, we believe that larger prospective studies should be performed in hypothyroid women to clarify a potential association between hypothyroidism and breast cancer.

\section{Declaration of interest}

The authors declare that there is no conflict of interest that could be perceived as prejudicing the impartiality of the review reported.

\section{Funding}

This review did not receive any specific grant from any funding agency in the public, commercial or not-for-profit sector.

\section{Author contribution statement}

A G Angelousi had the editorship of the manuscript, performed most of the statistical analysis and edited most of the manuscript. V K Anagnostou edited most of the manuscript. M K Stamatakos performed part of the literature search and edited part of the manuscript. G A Georgiopoulos edited part of the statistical analysis. K C Kontzoglou had the original idea for this article and edited part of the manuscript. All authors have read and approved the final manuscript.

\section{References}

1 National Statistics - UK Government Census, 2001. http://www. ons.gov.uk/ons/index.html

2 Cancer Facts and Figures 2004. American Cancer Society, 2004. http://www.cancer.org/acs/groups/content

3 Canadian Cancer Statistics 2004. National Cancer Institute of Canada, 2004. http://www.cancer.ca

4 Beatson GT. On the treatment of inoperable cases of carcinoma of the mamma: suggestions for a new method of treatment with illustrative cases. Lancet 19862 104-107.

5 Ellerker AG. Thyroid disorders and breast cancer: a causal connection? Medical Press 1956235 280-282.

6 Loeser AA. A new therapy for the prevention of post-operative recurrences in genital and breast cancer. A six years study of prophylactic thyroid treatment. BMJ 19542 1380-1383. (doi:10.1136/bmj.2.4901.1380)

7 Goldmann MB, Monson RR \& Maloof F. Cancer mortality in women with thyroid disease. Cancer Research $1990 \mathbf{5 0}$ 2281-2289.

8 Smyth PP. The thyroid and breast cancer: a significant association? Annals of Medicine 199729 189-191. (doi:10.3109/078538997 08999335)
9 Fiore E, Giustarini E, Mammoli C, Fragomeni F, Campani D, Muller I, Pinchera A \& Giani C. Favorable predictive value of thyroid autoimmunity in high aggressive breast cancer. Journal of Endocrinological Investigation 200730 734-738.

10 Giustarini E, Pinchera A, Fierabracci P, Roncella M, Fustaino L, Mammoli C \& Giani C. Thyroid autoimmunity in patients with malignant and benign breast diseases before surgery. European Journal of Endocrinology 2006154 645-649. (doi:10.1530/eje.1. 02108)

11 Smyth PP, Shering SG, Kilbane MT, Murray MJ, McDermott EW, Smith DF \& O'Higgins NJ. Serum thyroid peroxidase autoantibodies, thyroid volume, and outcome in breast carcinoma. Journal of Clinical Endocrinology and Metabolism 1998 83 2711-2716. (doi:10.1210/jc.83.8.2711)

12 Dai G, Levy O \& Carrasco N. Cloning and characterisation of the thyroid iodide symporter. Nature 1996379 458-460. (doi:10. 1038/379458a0)

13 Giani C, Fierabracci P, Bonacci R, Gigliotti A, Campani D, De Negri F, Cecchetti D, Martino E \& Pinchera A. Relationship between breast cancer and thyroid disease: relevance of autoimmune thyroid disorders in breast malignancy. Journal of Clinical Endocrinology and Metabolism 199681 990-994. (doi:10.1210/ jc.81.3.990)

14 Chung JK. Sodium iodide symporter: its role in nuclear medicine. Journal of Nuclear Medicine 200243 1188-2000.

15 Turken O, Narin Y, DemIrbas S, Onde ME, Sayan O, KandemIr EG, YaylacI M \& Ozturk A. Breast cancer in association with thyroid disorders. Breast Cancer Research 20035 110-113. (doi:10.1186/ bcr609)

16 Fierabracci P, Pinchera A, Campani D, Pollina LE, Giustarini E \& Giani C. Association between breast cancer and autoimmune thyroid disorders: no increase of lymphocytic infiltrates in breast malignant tissues. Journal of Endocrinological Investigation 200629 248-251.

17 Kilbane MT, Ajjan RA, Weetman AP, Dwyer R, McDermott EW, O'Higgins NJ \& Smyth PP. Tissue iodine content and serummediated 125I uptake-blocking activity in breast cancer. Journal of Clinical Endocrinology and Metabolism 200085 1245-1250. (doi:10.1210/jc.85.3.1245)

18 Sandhu MK, Brezden-Masley C, Lipscombe LL, Zagorski B \& Booth GL. Autoimmune hypothyroidism and breast cancer in the elderly. Breast Cancer Research and Treatment 2009115 635-641. (doi:10.1007/s10549-008-0104-4)

19 González-Sancho JM, García V, Bonilla F \& Muñoz A. Thyroid hormone receptors/THR genes in human cancer. Cancer Letters 2003192 121-132. (doi:10.1016/S0304-3835 (02)00614-6)

20 Conde I, Paniagua R, Zamora J, Blánquez MJ, Fraile B, Ruiz A \& Arenas MI. Influence of thyroid hormone receptors on breast cancer cell proliferation. Annals of Oncology 200617 60-64. (doi:10.1093/annonc/mdj040)

21 Vorherr H. Thyroid disease in relation to breast cancer. Klinische Wochenschrift 197856 1139-1145. (doi:10.1007/BF01476857)

22 Shah NM, Eskin BA, Krouse TB \& Sparks CE. Iodoprotein formation by rat mammary glands during pregnancy and early postpartum period. Proceedings of the Society for Experimental Biology and Medicine 1986181 443-449.

23 Arafah BM. Increased need for thyroxine in women with hypothyroidism during estrogen therapy. New England Journal of Medicine 2001344 1743-1749. (doi:10.1056/NEJM20010607 3442302)

24 Kapdi CC \& Wolfe JN. Breast cancer. Relationship to thyroid supplements for hypothyroidism. Journal of the American Medical Association $1976 \quad 6$ 1124-1127. (doi:10.1001/jama.236.10. 1124)

25 Adami HO, Rimsten A, Thorén L, Vegelius J \& Wide L. Thyroid disease and function in breast cancer patients and nonhospitalized controls evaluated by determination of TSH, $\mathrm{T}_{3}, \mathrm{rT}_{3}$ and $\mathrm{T}_{4}$ levels in serum. Acta Chirurgica Scandinavica 1978144 89-97. 
26 Kalache A, Vessey MP \& McPherson K. Thyroid disease and breast cancer findings in a large case-control study. British Journal of Surgery 1982 69 434-435. (doi:10.1002/bjs.1800690731)

27 Brinton LA, Hoffman DA, Hoover R \& Fraumeni JF Jr. Relationship of thyroid disease and use of thyroid supplements to breast cancer risk. Journal of Chronic Diseases 198437 877-893. (doi:10.1016/ 0021-9681(84)90062-6)

28 Goldmann MB, Monson RR \& Maloof F. Benign thyroid diseases and the risk of death from breast cancer. Oncology $1992 \mathbf{4 9}$ 461-466. (doi:10.1159/000227093)

29 Shering SG, Zbar AP, Moriarty M, McDermott EW, O’Higgins NJ \& Smyth PP. Thyroid disorders and breast cancer. European Journal of Cancer Prevention $19965504-506$.

30 Smyth PP, Smith DF, McDermott EW, Murray MJ, Geraghty JG \& O'Higgins NJ. A direct relationship between thyroid enlargement and breast cancer. Journal of Clinical Endocrinology and Metabolism 199681 937-941. (doi:10.1210/jc.81.3.937)

31 Simon MS, Tang MT, Bernstein L, Norman SA, Weiss L, Burkman RT, Daling JR, Deapen D, Folger SG, Malone K, Marchbanks PA, McDonald JA, Strom BL, Wilson HG \& Spirtas R. Do thyroid disorders increase the risk of breast cancer? Cancer Epidemiology, Biomarkers $\mathcal{E}$ Prevention 200211 1574-1578.

32 Cristofanilli M, Yamamura Y, Kau SW, Bevers T, Strom S, Patangan M, Hsu L, Krishnamurthy S, Theriault RL \& Hortobagyi GN. Thyroid hormone and breast carcinoma. Primary hypothyroidism is associated with a reduced incidence of primary breast carcinoma. Cancer 2005103 1122-1128. (doi:10.1002/cncr.20881)

33 Kuijpens JL, Nyklíctek I, Louwman MW, Weetman TA, Pop VJ \& Coebergh JW. Hypothyroidism might be related to breast cancer in post-menopausal women. Thyroid 200515 1253-1259. (doi:10. 1089/thy.2005.15.1253)

34 Ditsch N, Liebhardt S, Von Koch F, Lenhard M, Vogeser M, Spitzweg C, Gallwas J \& Toth B. Thyroid function in breast cancer patients. Anticancer Research $2010301713-1717$.

35 Bradburn MJ, Deeks JJ \& Altman DG. Sbe24: metan - an alternative meta-analysis command. Stata Technical Bulletin $1998 \mathbf{4 4} 15$.

36 DerSimonian R \& Laird N. Meta analysis in clinical trials. Controlled Clinical Trials 19867 177-188. (doi:10.1016/01972456(86)90046-2)
37 Higgins JP, Thompson SG, Deeks JJ \& Altman DG. Measuring inconsistency in meta-analyses. BMJ $2003 \mathbf{3 2 7} 557-560$. (doi:10.1136/bmj.327.7414.557)

38 Stroup DF, Berlin JA, Morton SC, Olkin I, Williamson GD, Rennie D, Moher D, Becker BJ, Sipe TA \& Thacker SB. Meta-analysis of observational studies in epidemiology: a proposal for reporting. Meta-analysis Of Observational Studies in Epidemiology (MOOSE) group. Journal of the American Medical Association 2000283 2008-2012. (doi:10.1001/jama.283.15.2008)

39 Funahashi H, Imai T, Tanaka Y, Tobinaga J, Wada M, Morita T, Yamada F, Tsukamura K, Oiwa M, Kikumori T, Narita T \& Takagi H. Suppressive effect of iodine on DMBA induced breast tumour growth in the rat. Journal of Surgical Oncology $1996 \mathbf{6 1}$ 209-213. (doi:10.1002/(SICI)1096-9098(199603)61:3<209:: AID-JSO9>3.0.CO;2-F)

40 Eskin BA, Grotowski CE, Connolly CP \& Ghent W. Different tissue responses for iodine and iodide in rat thyroid and mammary glands. Biological Trace Element Research 1995 49 9-19. (doi:10. 1007/BF02788999)

41 Silva JM, Dominguez G, Gonzalez-Sancho JM, García JM, Silva J, García-Andrade C, Navarro A, Muñoz A \& Bonilla F. Expression of thyroid hormone receptor/erbA genes is altered inhuman breast cancer. Oncogene 200221 4307-4316. (doi:10.1038/sj.onc. 1205534)

42 Mustacchi P \& Greenspan F. Thyroid supplementation for hypothyroidism. An latrogenic cause of breast cancer? Journal of the American Medical Association 1977237 1446-1447. (doi:10. 1001/jama.237.14.1446)

43 Shapiro S, Slone D, Kaufman DW, Rosenberg L, Miettinen OS, Stolley PD, Knapp RC, Leavitt T Jr, Watring WG, Rosenshein NB \& Schottenfeld D. Use of thyroid supplements in relation to the risk of breast cancer. Journal of the American Medical Association 1980 244 1685-1687. (doi:10.1001/jama.244.15.1685)

Received 27 September 2011

Revised version received 20 October 2011

Accepted 24 October 2011 